\title{
Producción de briquetas a partir de raquis residual de la palma africana para las comunidades alfareras del norte del Cesar (Colombia)
}

\author{
Marlon Bastidas-Barranco ${ }^{1 *}$, Robert Valera-Restrepo ${ }^{2}$ y Dario Serrano-Florez ${ }^{1}$ \\ (1) Facultad de Ingenierías, Ingeniería Mecánica, Grupo DESTACAR, Universidad de La Guajira, Colombia \\ (2) Facultad de Ingenierías y Tecnologías, Ingeniería Agroindustrial, Grupo GEAB, Universidad Popular del Cesar, \\ Colombia (correo-e: marlonjoseb@uniguajira.edu.co, robertvalera@unicesar.edu.co, dserrano@uniguajira.edu.co) \\ * Autor a quien debe ser dirigida la correspondencia
}

Recibido Jun. 20, 2021; Aceptado Ago. 18, 2021; Versión final Oct. 18, 2021, Publicado Feb. 2022

\begin{abstract}
Resumen
Este trabajo tiene como objetivo analizar las condiciones térmicas, ambientales y económicas mínimas para utilizar briquetas de raquis de la palma de aceite como sustituto de la leña en comunidades campesinas de la zona alfarera en el norte del departamento del Cesar, Colombia. Se plantea la construcción de una prensa sencilla para elaboración de briquetas. Se caracteriza el raquis físico-químicamente y biológicamente y se comparan los contenidos energéticos de la briqueta y la leña nativa de la zona. Los resultados muestran que la mayoría de los parámetros evaluados de las briquetas son similares a los de la leña nativa, siendo la relación másica de $\mathrm{CO} / \mathrm{CO}_{2}$ superior en la briqueta que en la cocción de ladrillos. El costo de una briqueta de $90 \mathrm{~g}$ es de $\$ 5.5$ pesos colombianos (COP). Se concluye que el reemplazo de la leña nativa por briquetas de raquis es un sustituto alternativo para aminorar la deforestación en la zona alfarera del norte del departamento del Cesar.
\end{abstract}

Palabras clave: briquetas; energía; leña; raquis; relación $\mathrm{CO} / \mathrm{CO}_{2}$

\section{Briquettes made from residual African palm rachis for pottery communities from northern Cesar (Colombia)}

\begin{abstract}
This study aims to determine and assess the minimum thermal, environmental, and economic conditions required to use oil palm rachis briquettes as a substitute for firewood in rural pottery communities from northern Cesar, Colombia. The construction of a simple press for briquetting is presented. The physicochemical and biological characteristics of rachis are determined and the energy contents of briquettes and native firewood are compared. The results show that briquettes and native firewood are similar in most of the parameters examined, but for briquettes the mass $\mathrm{CO} / \mathrm{CO}_{2}$ ratio is higher than that of native firewood when used under the same conditions of energy expenditure during brick firing. The cost of a 90 gram briquette is $\$ 5.5$ Colombian pesos (COP). It is concluded that substituting native firewood for rachis briquettes is a viable option to reduce deforestation in the pottery area of northern Cesar.
\end{abstract}

Keywords: briquettes; energy; firewood; rachis; $\mathrm{CO} / \mathrm{CO}_{2}$ ratio 


\section{INTRODUCCIÓN}

El departamento del Cesar ha sido habitualmente de vocación agrícola; sin embargo, a partir de la década de los 80 s, la explotación de carbón en minas a cielo abierto y la situación social de orden público en el territorio han ido desplazando los cultivos tradicionales por actividades alternas entre las que se destaca la industrialización de cultivos de palma africana, llegando a producirse en Colombia 47 millones de toneladas de aceite en 2010, situación que encumbró al país hasta ocupar el quinto lugar en producción de aceite de palma a nivel mundial (Rey, 2013). Con la incursión masiva e industrial de los cultivos de palma en el país, siendo el Valle del Cesar ubicado al norte de Colombia, uno de los grandes focos de cultivo, condujo a los campesinos desarrollar actividades alternas que requerían de poca escolaridad, destacándose entre otras, el plegamiento a la producción de ladrillos artesanales en grupos de familias (Ávila et al., 2015).

La situación planteada generó dos grandes problemáticas; por un lado, una creciente generación de residuos biomásicos derivados de la industria aceitera, estimándose para el Cesar cerca de unas 130 mil toneladas para el 2006 (Quintero et al., 2014); por otro lado, el aumento de familias dedicadas a la producción de ladrillos cuyo principal energético es el uso de la leña nativa de la zona, que además es utilizada para la cocción de alimentos, propiciando una actividad que conlleva a una marcada deforestación del territorio, la cual se reporta con datos apreciables de cerca de 1100 Ha deforestadas por año (Corporación Ambiental Empresarial, 2016).

Varios esfuerzos se han hecho para aprovechar los residuos biomásicos de la industria de la palma africana, siendo el uso como energéticos en los procesos de producción del aceite el de mayor aplicación; sin embargo, las cantidades requeridas son mínimas frente a los grandes volúmenes de residuos generados, por tanto, no es una solución de fondo a pesar que hay una sustitución de combustibles fósiles y, por ende, una disminución de los gases de efecto invernadero (GEI). En los últimos años las investigaciones referidas a la generación energética derivada de la biomasa han tomado gran interés (Ribó-Pérez et al., 2021; Hernández et al., 2017; Cabrera et al., 2011), ya que se ha constituido como un importante recurso renovable que posee propiedades interesantes como la recirculación ecológica de los GEl y la reconversión de un residuo en recurso energético con bajos costos de producción.

Uno de las principales variantes del uso energético de la biomasa residual es la preparación de briquetas, debido a que es una forma eficiente y sustentable de aprovechar estos residuos (Mitchual et al., 2014). Las briquetas se utilizan como alternativa energética para sustituir el carbón mineral y la leña, los primeros para reducir las emisiones de GEI y los segundos para evitar la deforestación y mejorar los rendimientos energéticos; en este sentido, varios trabajos se han dedicado a mostrar diversos materiales vegetales, según la vocación agrícola de las regiones, promisorios en la fabricación de briquetas como sustitutos de combustibles fósiles.

Se ha demostrado que es posible producir briquetas duraderas con baja compactación a partir de residuos de madera mezclados con residuos de la mazorca (Mitchual et al., 2014; Wilaipon, 2007), también se han utilizado residuos vegetales con altos contenidos carbonosos en diversas mezclas, entre otras la aceituna con residuo de la industria papelera (Yaman et al., 2007, Khlifi et al., 2020), la cáscara de coco con papel usado (Olorunnisola, 2007). Por otra parte, la elaboración de las briquetas requiere de tratamientos térmicos y mecánicos que permiten obtener la consistencia y las características de un combustible capaz de sustituir los fósiles y la leña, en este sentido, se muestran trabajos en los que se estudian los factores que afectan la resistencia y la durabilidad de los productos de la biomasa densificada en (Kaliyan and Vance, 2009) y el potencial energético contenido en la mazorca de maíz, con relación a su productividad, determinando experimentalmente el efecto de la relación de aglutinante de melaza y la presión de briqueteado sobre la densidad de briquetas elaboradas a partir de este residuo (Wilaipon, 2007).

Con lo anterior se muestra que los trabajos elaborados hasta la fecha se han encargado de evaluar las características de materiales vegetales y los métodos necesarios para obtener briquetas eficientes; sin embargo, se aprecia que se requiere de procesos complejos de secado, densificación y mezclas con aditivos químicos y biológicos para alcanzar la consistencia adecuada de la briqueta. Por otra parte, los métodos de evaluación del desempeño de la briqueta tienen el rigor relacionado con la misma complejidad que lleva la caracterización del material vegetal residual, el proceso de elaboración y la combustión de la briqueta.

Teniendo en cuenta que por lo general este tipo de biocombustible tiene como destino familias campesinas, es preferible un método sencillo que pueda ser asimilado por la población que requiere su uso, para que se vuelva una alternativa importante en la sustitución de combustibles fósiles y la leña. El objetivo de este trabajo es aportar una alternativa metodológica real para que los campesinos fabriquen su propio combustible en forma de briquetas con los residuos generados por la industria aceitera de la zona. La metodología propone el uso de recursos mínimos que estén al alcance de los campesinos, de tal manera que las propiedades energéticas de las briquetas sean comparables a la leña que están acostumbrados a utilizar, tanto en los procesos de producción de ladrillos. 
Esta estrategia aporta una opción ambiental para destinar un uso a los residuos de la industria aceitera de la zona de estudio, de tal manera que se pueda construir una alianza en la que se pueda proporcionar dichos residuos vegetales bajo ciertas condiciones de preparación para que las familias alfareras construyan briquetas artesanales y deje de utilizar leña en un esfuerzo de mejorar las condiciones de los ecosistemas, a la preservación y conservación de la vegetación boscosa nativa, a la disminución de las enfermedades producidas por el humo y gases de combustión incompleta, a la mitigación de las emisiones generadas a la atmosfera y aportando a las estrategias en contra del cambio climático mediante la producción limpia.

\section{MATERIALES Y MÉTODOS}

Este trabajo se llevó a cabo al norte del departamento del Cesar, Colombia en la zona de cultivo de palma cercana a los pequeños yacimientos alfareros intervenidos por familias campesinas, con la participación de los grupos de investigación GEAB y DESTACAR, de las Universidades del Cesar y La Guajira, respectivamente. Se tomaron muestras de residuos del cultivo de palma africana una vez extraído el aceite, específicamente racimos vacíos (raquis), y de leña nativa de la zona, frecuentemente utilizada en labores diarias por las 53 familias campesinas conformadas por 191 personas en las que laboran tan solo el 34\%, de las cuales se dedican a la alfarería el 85,3\% (Ávila et al., 2015). Los pasos metodológicos se describen a continuación.

\section{Recolección y preparación del material biomásico}

Se tomaron $10 \mathrm{~kg}$ de raquis como materia prima en la elaboración de las briquetas, el cual es desechado por las plantas extractoras de aceite del Grupo Empresarial Oleoflores. De igual manera, se tomaron $10 \mathrm{~kg}$ de leña nativa tipo Brasil (Haematoxylum brasilete), manteniendo las condiciones de uso de los campesinos, es decir, que la combustión del material se hace en un escenario similar al de las familias campesinas. Las muestras de raquis se sometieron a un proceso de secado natural durante 72 horas para garantizar el secado adecuado que permita la reducción del tamaño de partícula mediante un molino de martillo. El tamaño de partícula del raquis entregado del proceso de extracción de aceite está entre 200 a $280 \mathrm{~mm}$ de longitud de filamentos de $1 \mathrm{~mm}$ de radio aproximadamente. Para la leña, sólo es necesario el proceso de secado, el cual se establece con el mismo tiempo utilizado para el raquis.

\section{Construcción de prensa manual}

Para la compactación del material se construyó una prensa artesanal con material disponible para las familias campesinas. Los moldes se hicieron con tubos de PVC de diámetro de $50.80 \mathrm{~mm}$, largo de $150 \mathrm{~mm}$ y un émbolo de madera ajustado al orifico del tubo de PVC con el que se ejerce presión a la al raquis triturado, tal como se muestra en la Fig. 1a. En el interior del molde se incrustó una varilla $4.7 \mathrm{~mm}$ concéntrico al tubo de PVC (ver Fig. 1b) para obtener una briqueta con un orificio en el centro que permita una buena solidificación y mayor rendimiento de combustión. La prensa se construyó para ser operada con la fuerza de una persona.

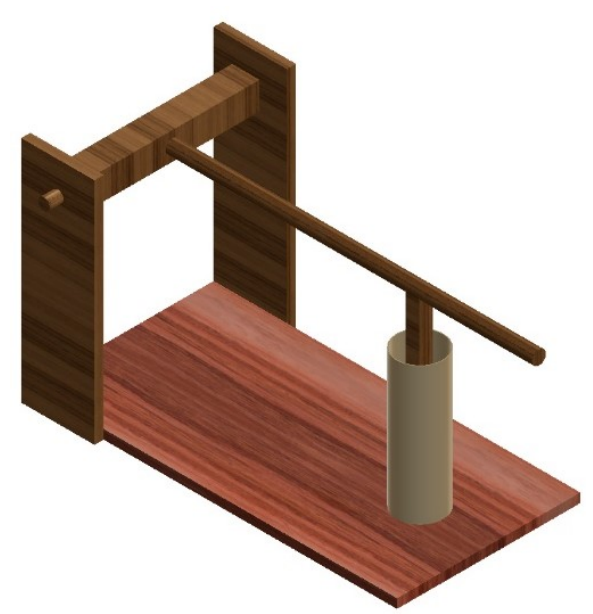

a)

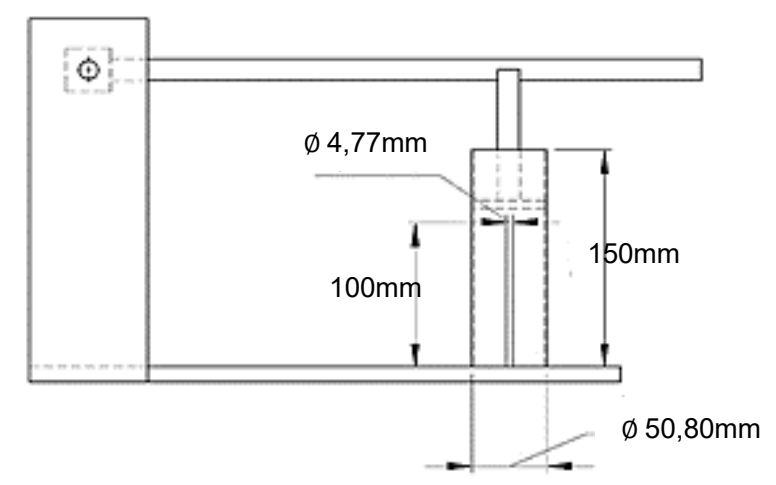

b)

Fig. 1. Prensa utilizada en la compactación de la mezcla, para la obtención del prototipo de briqueta. a) prensa artesanal para densificación de raquis; b) diseño de la prensa artesanal.

\section{Preparación y mezcla del aglomerante}

El aglomerante se preparó con extracto o almidón de yuca, el cual fue mezclado a proporciones de 15 y $20 \%$ p/p con el raquis fragmentado, con el fin de obtener una mezcla raquis-aglomerante consistente, que permita 
mantener una estructura fuerte para el almacenamiento y un rendimiento energético aproximado al obtenido de la leña (Acosta et al., 2017). Previo al mezclado, el aglomerante se disolvió en $150 \mathrm{ml}$ de agua destilada con agitación constante para evitar la formación de grumos, luego se calentó durante 10 minutos en un baño de agua a $100^{\circ} \mathrm{C}$ hasta formar una pasta; finalmente se pesó la cantidad de raquis triturado con la proporción definida de aglutinante manualmente hasta obtener una mezcla homogénea húmeda (Aransiola et al., 2019).

\section{Producción de briquetas}

La mezcla obtenida es introducida hasta el tope del tubo PVC la prensa, a la cual se le hace presión manual, tal como se muestra en la Fig. 1a, hasta alcanzar la altura entre 100 y $102 \mathrm{~mm}$, conservando el radio y orificio concéntrico detallado en el aparte de la construcción de la prensa. Una vez elaborada la briqueta manual es llevada a un proceso de secado natural por 30 días para estimar cambios en la densidad del material (Aransiola et al., 2019.

\section{Caracterización del raquis, las briquetas y la leña nativa}

Se hizo análisis del raquis por repetición del contenido de humedad (ASTM D3173-11), cenizas (ASTM D317412) celulosa y hemicelulosa (ASTM D1109-84) y lignina (ASTM D1106-96) y el poder calorífico (PC); las propiedades como humedad, cenizas y PC se compararon con los obtenidos de la leña nativa. Para las briquetas obtenidas a diferentes concentraciones de aglomerante se determinó la humedad, la densidad de compresión, la densidad de relajación después de 30 días de la elaboración, según lo recomendado por (Aransiola et al., 2019), como se observa en la Ec. 1. Adicionalmente se hizo un contenido de cenizas, poder calorífico, la fuerza de compresión hecha por un hombre promedio y la resistencia a la compresión para determinar la capacidad de almacenamiento.

$$
\rho=\frac{m}{V}=\frac{m}{\pi \cdot h \cdot\left(r_{e}^{2}-r_{i}^{2}\right)}
$$

\section{Análisis térmico, ambiental y económico}

Para el análisis térmico y ambiental se siguió lo sugerido por (Aristizábal, 2010), simplemente tomando un litro de agua utilizando estufas tradicionales con leña nativa y las briquetas producidas, pero en este caso con seguimiento de la temperatura, el tiempo trascurrido y el consumo de combustible para el calentamiento de un litro de agua durante el mismo tiempo bajo condiciones similares. Para el análisis térmico se siguió la Ec. 2, donde $\mathrm{Q}$ es el calor entregado en el proceso, PC y $\mathrm{m}$ es el poder calorífico y la masa gastada del combustible, respectivamente e i es el tipo de combustible (leña o briqueta); en la Ec. 3, $Q_{w}$ es el calor recibido por el agua, Cp es el calor específico del agua, $m_{w}$ es la masa de agua, $m_{e}$ es la masa de agua evaporada, $\Delta T$ es el cambio de temperatura del agua durante el proceso y $\lambda_{\mathrm{w}}$ es el calor latente de evaporación a presión atmosférica al nivel del mar; en la Ec. 4, se detalla la eficiencia $\eta$ de la estufa. Para las constantes $\mathrm{Cp}$ y $\lambda_{\mathrm{w}}$ se toman valores de 4.186 $\mathrm{kJ} / \mathrm{kg}{ }^{\circ} \mathrm{C}$ y $2257 \mathrm{~kJ} / \mathrm{kg}$ a $97^{\circ} \mathrm{C}$, respectivamente.

$$
\begin{aligned}
& \mathrm{Q}_{\mathrm{i}}=\mathrm{m}_{\mathrm{i}} \cdot P C_{\mathrm{i}} \\
& \mathrm{Q}_{\mathrm{w}}=\mathrm{m}_{\mathrm{w}} \cdot \mathrm{Cp} \cdot \Delta \mathrm{T}+\mathrm{m}_{\mathrm{e}} \cdot \lambda_{\mathrm{w}} \\
& \eta=\frac{\mathrm{Q}_{\mathrm{w}}}{\mathrm{Q}_{\mathrm{i}}} * 100
\end{aligned}
$$

Para el análisis ambiental, se toman muestras de gases durante el proceso de calentamiento y se determina las concentraciones de $\mathrm{CO}_{2}$ y $\mathrm{CO}$ y se comparan emisiones de las briquetas y la leña bajo las mismas condiciones y con lo presentado en la literatura. Los gases se tomaron en bolsas colectoras de gas mediante una bomba de vacío y se hicieron pasar por un analizador de gases 3000 Micro GC portátil, con tres repeticiones para disminuir la incertidumbre de la información obtenido, con unas desviaciones de alrededor del $1 \%$.

Es importante establecer la favorabilidad económica para los alfareros y familias campesinas la elaboración de briquetas con respecto a los costos de la leña; por lo tanto, se determinó por información primaria como se adquiere la leña para la cocción de ladrillos y el consumo diario de los hogares, respectivamente. Para los alfareros la leña se permuta por ladrillos, en una relación de carga $n_{T}=3000$ mil ladrillos por carga de leña con la cual se pueden producir en promedio $\mathrm{N}_{\mathrm{T}}=11250$ ladrillos en hornos pampa de baja eficiencia (Quintero et al, 2018); los 3000 ladrillos tienen un costo total $C_{T}=210$ mil COP en el mercado local. Por otra parte, los hogares tienen un consumo de $4 \mathrm{~kg}$ diarios de leña en promedio, las cuales toman de los alrededores de las viviendas. 
Se calcula la cantidad energía requerida por cada ladrillo $E_{L}$, con base en el calor de cocción de los ladrillos $\mathrm{Q}_{\mathrm{L}}=1396.6 \mathrm{kBTU} /$ ton de ladrillos, correspondiente a $1473.5 \mathrm{~kJ} / \mathrm{kg}$ de ladrillos (García et al., 2013) y la masa promedio de un ladrillo $m_{L}=4.7 \mathrm{~kg}$ (Quintero et al, 2018) (Ec. 3); la masa requerida de leña nativa $m_{\mathrm{ln}}$ se calcula de la relación de energía por ladrillo y el poder calorífico superior de la leña nativa $P C S_{l n}=17539 \mathrm{~kJ} / \mathrm{kg}(E c$. 6 ), de igual manera la masa de briqueta requerida por ladrillo $\mathrm{m}_{B}$ con el poder calorífico superior de la briqueta $\mathrm{PCS}_{\mathrm{B}}$ $=18071 \mathrm{~kJ} / \mathrm{kg}$ (Ec. 7 ) y el costo de un ladrillo cocido se obtiene del cociente entre el costo total de carga y los ladrillos producidos menos los canjeados por una carga de leña $\mathrm{N}_{\mathrm{T}}-\mathrm{n}_{\mathrm{T}}$ (Ec. 8).

$$
\begin{aligned}
& E_{L}=Q_{L} \cdot m_{L} \\
& m_{l n}=\frac{E_{L}}{P C S_{l n}} \\
& m_{B}=\frac{E_{L}}{P C S_{B}} \\
& c_{L}=\frac{C_{T}}{N_{T}-n_{T}}
\end{aligned}
$$

\section{RESULTADOS Y DISCUSIÓN}

Los resultados obtenidos se tomaron de raquis después del secado con una reducción del tamaño hasta 10 a $30 \mathrm{~mm}$ de longitud. Las propiedades del raquis, las briquetas producidas y la leña son obtenidas para apreciar la transformación del material, la desviación de los resultados finales con respecto a los reportados por otros autores y una comparación de contenido y uso energético entre las briquetas y la leña nativa.

\section{Características fisicoquímicas y biológicas del raquis}

Es importante caracterizar la biomasa para la producción de briquetas, debido a que los contenidos de lignina son variables (ver Tabla 1), hay una diferencia apreciable de este valor entre lo encontrado y los autores (Acosta et al., 2017) y (Moreno, 2015). Aunque el raquis tomado de este trabajo es de la misma región que el autor (Acosta et al., 2017), el autor (Moreno, 2015) no menciona el origen de las muestras. De cualquier manera, los porcentajes de lignina son altos para el raquis de la zona de estudio con respecto a otros materiales, pero estos valores están por debajo al 45\% recomendado por (Acosta et al., 2017) para la formación de briquetas consistentes; por lo tanto, es necesario un aditivo para alcanzar compactación.

Tabla 1: Propiedades fisicoquímicas y biológicas del raquis

\begin{tabular}{|l|c|l|}
\hline Ensayo & Resultados & \multicolumn{1}{c|}{ Resultados (Otros autores) } \\
\hline Humedad [\%] & 7.54 & $\begin{array}{l}7.39 \text { (Husain et al., 2002), 12.0 (Zhang and Guo, 2014), 7.21 (Acosta et al., } \\
2017)\end{array}$ \\
\hline Cenizas [\% bs] & 6.98 & $\begin{array}{l}7.3 \text { (Husain et al., 2002), 2.41 (Zhang and Guo, 2014), 7.58 (Acosta et al., } \\
2017)\end{array}$ \\
\hline Material Volátil [\% bs] & 65.23 & 75.7 (Husain et al., 2002), 69.14 (Acosta et al., 2017) \\
\hline Carbono Fijo [\% bs] & 17.02 & 17 (Husain et al., 2002), 16.07 (Acosta et al., 2017) \\
\hline PCS [kJ/kg] & 17533 & $\begin{array}{l}17823 \text { (Husain et al., 2002), 17000 (Zhang and Guo, 2014), 17140 (Acosta et } \\
\text { al., 2017) }\end{array}$ \\
\hline PCl [kJ/kg] & 16110 & 15870 (Acosta et al., 2017), 16823 (MME, 2008) \\
\hline $\begin{array}{l}\text { Celulosa y } \\
\text { hemicelulosa [\% bs] }\end{array}$ & 60.52 & 57.64 (Acosta et al., 2017), 19 (Moreno, 2015). \\
\hline Lignina [\% bs] & 15.30 & 22.49 (Acosta et al., 2017), 8.8 (Moreno, 2015). \\
\hline
\end{tabular}

En los análisis próximos, la humedad se midió mediante un analizador de humedad (OAHUS MB45) calibrado bajo la normativa ASAE s 358.2, llevando la muestra a $103^{\circ} \mathrm{C}$ por un tiempo específico, se mide la diferencia de masas, donde la masa eliminada corresponde al agua dentro de las partículas de la materia. El valor de poder calorífico (volumen constante) en base seca fue determinado acorde a la norma ASTM 5142. La combustión se realizó empleando una bomba calorimétrica modelo AC-350 marca LECO a una presión de $3102.66 \mathrm{kPa}(450 \mathrm{psi})$. El procedimiento utilizado para la determinación del rendimiento en cenizas de la biomasa es el NREL/TP-510-42622. Se calcina 1 gramo de muestra de madera seca a $575{ }^{\circ} \mathrm{C}$ durante 24 horas. Se dejan enfriar en un desecador y se pesan para determinar el porcentaje en cenizas. Por otro lado, el contenido en celulosa de la biomasa se ha determinado según el procedimiento TAPPI T 212, que consiste en tomar 1 gramo de muestra de holocelulosa que se coloca en un matraz de 100 mililitros en un baño de agua a $25^{\circ} \mathrm{C}$. Se añaden 10 mililitros de hidróxido sódico del $17.5 \%$, se agita y se deja reposar 5 minutos. Luego, se añaden 5 mililitros de hidróxido sódico al $17.5 \%$, se agita y se reposa 30 minutos. Se añaden finalmente 30 mililitros de agua, se agita y se deja reposar 1 hora. 
Por último, la cuantificación de la lignina se realiza según el procedimiento NREL/TP-510-42618. Se pesan $300.0 \pm 10.0$ miligramos de muestra en un tubo de presión tarado. Se añaden $3.00 \pm 0.01$ mililitros de ácido sulfúrico del $72 \%$ a cada tubo de presión. Se dejan los tubos de presión en un baño de agua a $30 \pm 3{ }^{\circ} \mathrm{C}$ e incubar la muestra durante 60 minutos. El material volátil se determina calentando la muestra en un crisol tapado durante siete minutos a $950^{\circ} \mathrm{C}$; a esta temperatura se lleva a cabo un proceso de descomposición de la materia orgánica por ruptura de enlaces químicos (pirolisis). La perdida en peso que sufre la muestra, una vez descontada su humedad, indica la cantidad de compuestos gaseosos producidos en la descomposición de la sustancia carbonosa. El Carbono fijo es la fracción de carbono residual que permanece luego de retirar de la muestra la humedad, las cenizas, y el material volátil.

\section{Obtención de briquetas al 15 y $20 \%$}

En la Fig. 2, se muestra la forma del prensado manual (a) para la compactación de las briquetas, cuyos resultados no son buenos para porcentajes de aglomerantes menores al $15 \%$ (b), pero cuando es del $20 \%$ se muestra una briqueta bien formada (c), ratificando lo planteado por (Acosta et al., 2017). Tanto el tamaño de la partícula del raquis, como la fuerza de compactación intervienen en la forma y resistencia de la briqueta; el hecho de hacer una briqueta con compactación manual y tamaño de partícula de $15 \mathrm{~mm}$ en promedio, son grandes desventajas frente a la elaboración de briquetas, que generalmente se elaboran con compactación mecánica y material pulverizado, tal como se presenta en la literatura. Sin embargo, un método simple de elaboración de briquetas como sustituto de la leña nativa, puede ser una solución inicial para la conservación de la vegetación de la zona a costa de afectar el rendimiento energético de la briqueta.

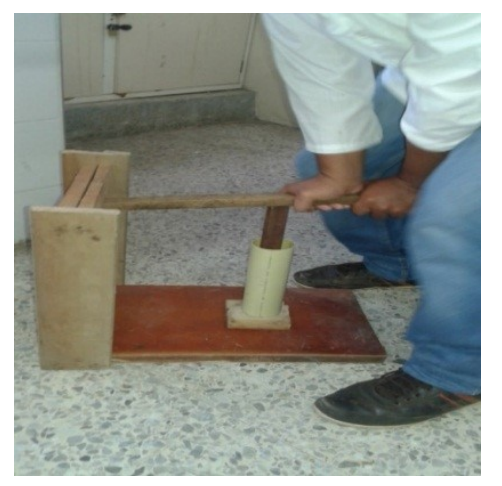

a)
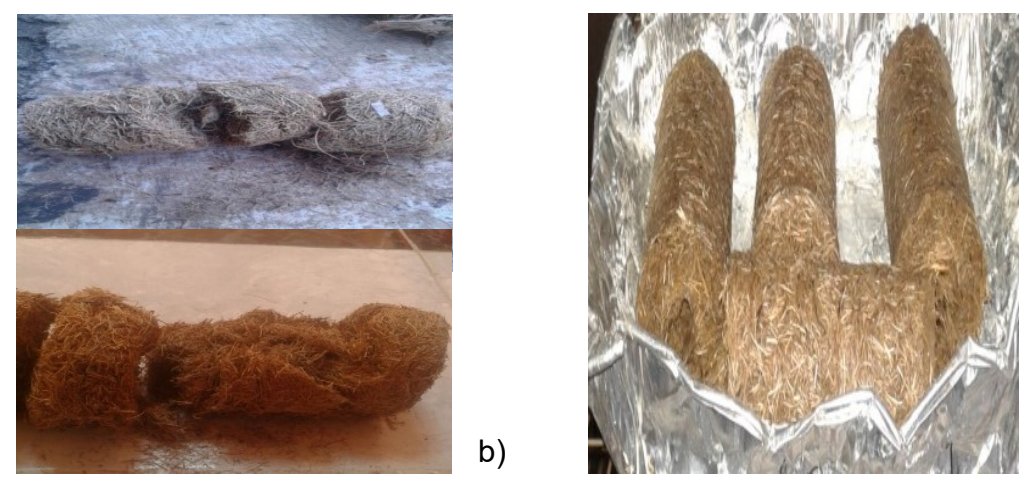

c)

Fig. 2. Proceso de preparación de Briquetas. a) Compactación de briquetas [10]; b) Briquetas obtenidas con aglomerante a $15 \%$ [10]; c) Briquetas obtenidas con aglomerante con $20 \%$ [10].

\section{Densidad comprimida y relajada}

En la Tabla 2, se muestran en las dos primeras filas los valores de la densidad de compresión con la aplicación de la fuerza de un humano que está entre 1.0 y $1.7 \mathrm{kPa}$ (Acosta et al., 2017) y la densidad de relajación de las briquetas con aglomerante al 15 y $20 \%$, comparadas con las presentadas por (Aransiola et al., 2019) en las tres últimas filas aplicadas a fuerzas de 50,100 y $150 \mathrm{kPa}$, respectivamente, con aglutinantes similares. Se debe tener en cuenta que la materia prima de las briquetas elaboradas por (Aransiola et al., 2019) es la tusa de maíz, mientras que este trabajo presenta briquetas a partir de raquis de palma africana.

Tabla 2: Densidad de compresión (DC) y relajación (DR) para briquetas al $15 \%$ y $20 \%$

\begin{tabular}{|c|c|c|c|}
\hline Presión [kPa] & Aglutinante [\%] & $D C\left[\mathrm{~kg} / \mathrm{m}^{3}\right]$ & $D R\left[\mathrm{~kg} / \mathrm{m}^{3}\right]$ \\
\hline \multirow{2}{*}{$1.0-1.7$} & 15 & 502.3 & 460.1 \\
\cline { 2 - 4 } & 20 & 539.2 & 484.4 \\
\hline 50 & 20 & 1239.83 & 896.42 \\
\hline 100 & 20 & 1306.28 & 925.84 \\
\hline 150 & 20 & 1393.32 & 962.67 \\
\hline
\end{tabular}

Para la compresión con la fuerza de un humano se obtienen densidades moderadas del material, pero se alcanza una buena consistencia al punto que soporta presiones en dirección vertical sin deformarse de $2.20 \mathrm{MPa}$ y en dirección horizontal sin deformarse de $1.85 \mathrm{MPa}$ (Acosta et al., 2017), frente a los 7.7 MPa y 0.34 de las briquetas elaboradas por (Nasrin et al., 2008), esto indica que, a pesar de tener compactación sin el rigor de las briquetas industriales, tienen una gran capacidad de soporte para el almacenamiento. 


\section{Análisis de la elaboración de briquetas del estudio}

Durante los pasos requeridos para la elaboración de briquetas se tuvo en cuenta aspectos ambientales, económicos y técnicos que son importante para determinar los aportes realizados por el campesino, las emisiones de gases debido a la combustión de la briqueta; en comparación con la leña y la oportunidad de reemplazar tecnologías que mejoren la eficiencia de la combustión. A continuación, se señalan las evaluaciones señaladas.

\section{Evaluación térmica}

Se presenta el análisis térmico donde se nota una pérdida de agua, necesaria para establecer el calor que entrega el combustible; por lo tanto, se hizo un proceso similar para determinar las variables implicada en dicho proceso, tanto para el uso de las briquetas como en la leña nativa con la finalidad de hacer un análisis de los detalles y comparar con resultados reportados en la literatura. En la Tabla 3, se puede apreciar que el calor aportado tanto por la briqueta como la leña son similares, aunque la leña nativa requirió un tiempo mayor con un aporte de calor adicional en la evaporación de agua. La eficiencia de la estufa tradicional es baja, debido a la capacidad calórica de los mismos. La eficiencia de la estufa es mayor al encontrado por (Sierra et al., 2014), que reporta para estufas nativas eficiencia del orden de $2.4 \%$ y por debajo de la eficiencia de las estufas mejoradas descritas por (Aristizábal, 2010) con rendimientos mayores al 14\% con respecto a las estufas tradicionales.

Tabla 3: Variables medidas para combustión de la briqueta y la leña nativa

\begin{tabular}{|c|c|c|}
\hline Variable & Briquetas & Leña nativa \\
\hline $\mathrm{Q}_{\mathrm{i}}(\mathrm{kJ})$ & 39276.18 & 42128.68 \\
\hline $\mathrm{Q}_{\mathrm{w}}(\mathrm{kJ})$ & 1750.39 & 1831.64 \\
\hline$\eta(\%)$ & 4.46 & 4.35 \\
\hline $\mathrm{m}_{\mathrm{i}}(\mathrm{kg})$ & 2.438 & 2.402 \\
\hline $\mathrm{m}_{\mathrm{e}}(\mathrm{kg})$ & 0.642 & 0.678 \\
\hline $\mathrm{t}(\mathrm{s})$ & 1140 & 1260 \\
\hline
\end{tabular}

\section{Evaluación ambiental}

Se hace un análisis de la relación de la masa de $\mathrm{CO}$ con respecto a la masa de $\mathrm{CO}_{2}$ de la briqueta y la leña nativa, como un indicador ambiental del uso de combustibles. Se aprecia en la Tabla 4, que la relación másica de $\mathrm{CO} / \mathrm{CO}_{2}$ es mayor para las briquetas que para la leña nativa en estufas campesinas, lo que indica que hay un factor del diseño de las briquetas que impide combustión completa en el lecho, lo cual se puede corregir con estufas eficientes, en las que se pueda controlar el flujo de aire y el confinamiento de los gases calientes a través de chimeneas con un tiempo de retención adecuado para la transferencia de calor; sin embargo, tanto para estufas campesinas como mejoradas, la leña tiende a tener mejor combustión, pero para estufas mejoradas la producción de CO está por debajo de los $0.01 \mathrm{~kg}$ por cada kg de $\mathrm{CO}_{2}$ generado en la combustión.

Tabla 4: Relación de emisiones de $\mathrm{CO} / \mathrm{CO}_{2}$ para las briquetas y la leña nativa

\begin{tabular}{|c|c|c|}
\hline Combustible & Relación $\mathrm{CO} / \mathrm{CO}_{2}[\mathrm{~kg} / \mathrm{kg}]$ & Autor \\
\hline Briquetas de raquis & 0.573 & \multirow[b]{2}{*}{ - } \\
\hline Leña nativa & 0.321 & \\
\hline $80 \%$ acícula $+20 \%$ papel & 0.075 & \multirow{6}{*}{ (Gamarra, 2010) } \\
\hline $40 \%$ papel $+60 \%$ aserrín & 0.054 & \\
\hline $20 \%$ papel $+50 \%$ pasto $+30 \%$ aserrín & 0.070 & \\
\hline $25 \%$ papel $+40 \%$ acícula $+35 \%$ aserrín & 0.062 & \\
\hline Leña de pino & 0.030 & \\
\hline $80 \%$ acícula $+20 \%$ papel & 0.075 & \\
\hline
\end{tabular}

\section{Evaluación económica}

De los balances se obtienen valores de energía, masa de leña nativa, masa de briquetas y costos por cada ladrillo producido con leña nativa; con lo que se obtienen $E_{L}=6925.5 \mathrm{~kJ}, m_{\ln }=0.395 \mathrm{~kg}, \mathrm{~m}_{\mathrm{B}}=0.421 \mathrm{~kg} \mathrm{y}$ $\mathrm{C}_{\mathrm{L}}=25.5 \mathrm{COP}$, respectivamente. Si se invierten los $210 \mathrm{mil}$ COP en la producción de briquetas en reemplazo de la leña nativa, el costo máximo que se debe pagar las briquetas sería de $60.57 \mathrm{COP} / \mathrm{kg}$, lo que significa que el costo de una briqueta de masa $90 \mathrm{~g}$ sería de 5.5 COP. Finalmente, la inversión anual en compra de las 353.3 toneladas de leña es de 22.8 Millones de COP; los cuales pueden ser invertidos para preparar 376.5 toneladas de briquetas con un aglomerante de almidón de yuca al $20 \%$. 


\section{CONCLUSIONES}

De acuerdo al trabajo presentado, a los resultados obtenidos y su análisis, se pueden plantear las siguientes conclusiones principales:

i) Una alternativa interesante para aminorar la deforestación en la zona alfarera del norte del departamento del Cesar - Colombia, sería el reemplazo de la leña nativa por briquetas elaboradas del raquis residual de la industria aceitera cercana, teniendo en cuenta que se pueden elaborar con recursos de fácil adquisición por las familias campesinas de dicha zona.

(ii) La briqueta elaborada requiere por lo menos de un $20 \%$ de aglomerante de almidón de yuca para que adquiera la consistencia adecuada para el transporte y almacenamiento, aplicando una fuerza de compresión realizada por un hombre, la cual es mucho menor a las empleadas por las máquinas usadas en la compactación de briquetas.

(iii) Considerando que las características físico-químicas de la briqueta y la leña nativa son similares, se determinó que cada kilogramo de leña para uso energético se puede reemplazar por $1.065 \mathrm{~kg}$ de briqueta al $20 \%$, esto quiere decir que son necesarios $0.85 \mathrm{~kg}$ de raquis, teniendo un indicador interesante para la utilidad de los residuos de la palma africana.

(iv) La combustión de la leña nativa y la briqueta en cocinas artesanales es de muy baja eficiencia, teniendo pérdidas de energía por encima del $90 \%$ y con una relación másica de $\mathrm{CO} / \mathrm{CO}_{2}$ por encima del $0.1 \mathrm{~kg} / \mathrm{kg}$, lo cual es muy alto si se compara con los reportes para cocinas mejoradas.

(v) El costo máximo de la elaboración de las briquetas de $90 \mathrm{~g}$, es de $5.5 \mathrm{COP}$, para no superar los costos de obtención de ladrillos con leña nativa; sin embargo, considerando los costos ambientales del uso de la leña, se puede pensar en un costo adicional si es necesario, destinado a la molienda del raquis en el sitio y transporte de las briquetas a los puntos de combustión.

\section{REFERENCIAS}

Acosta, E., Noriega, J., y Bastidas, M., Evaluación de briquetas elaboradas con raquis residual como alternativa energética al uso de la leña, Congreso Internacional de Energías Renovables (CIERG) - Memorias, Primera Edición, Servicio Nacional de Aprendizaje (SENA), 155-170, Riohacha, Colombia (2017).

Aransiola, E.F., Oyewusi, T.F., y otros dos autores, Effect of binder type, binder concentration and compacting pressure on some physical properties of carbonized corncob briquette, https://doi.org/10.1016/j.egyr.2019.07.011, Energy Reports, 5, 909918 (2019).

Aristizábal, J.D., Estufas mejoradas y bancos de leña: una alternativa de abastecimiento energético a nivel de finca para comunidades dependientes de los bosques de roble de la cordillera oriental, Revista Colombia Forestal, 13(2), 245-265 (2010).

Ávila, L.A., Molina, V.A., y Mendoza, M.L., Análisis socioeconómico de la población con asentamiento en la cantera el cielo situado en el corregimiento de Valencia de Jesús, Cesar, Colombia, VI Encuentro Nacional de Investigación Formativa Ingeniería Industrial Medellín - Memorias, Primera Edición, Editorial Universidad Pontificia Bolivariana, 5-22, Medellín, Colombia (2015).

Cabrera, G., Burbano, J.C, y García, J.I., Preliminary Analysis of Biomass Potentially Useful for Producing Biodiesel, DYNA, 78(170), 144-151 (2011).

Corporación Ambiental Empresarial, Plan Integral de Gestión de Cambio Climático Territorial del Cesar 2032, 1-161, Bogotá, Colombia (2016).

Gamarra, L.A., Fabricación y evaluación de eficiencia y emisiones de briquetas a base de residuos agrícolas como alternativa energética al uso de leña, Universidad Zamorano, Zamorano, Honduras (2010).

García-Ubaque, C.A., Vaca-Bohórquez, M.L., y Talero, G.F., Aprovechamiento de Biomasa Peletizada en el Sector Ladrillero en Bogotá-Colombia: Análisis Energético y Ambiental, http://dx.doi.org/10.4067/S0718-07642013000300013, Información Tecnológica, 24(3), 115-120 (2013).

Hernández, L.C., Forero, C.A., y Sierra-Vargas, F.E., Biomass Densification: A Review of the Current State-of-the-Art of the Pellet Market and Analysis of New Research Trends, http://dx.doi.org/10.18180/tecciencia.2017.23.10, Tecciencia, 12(23), 81-92 (2017)

Husain, Z., Zainac, Z., y Abdullah, Z., Briquetting of palm fiber and shell from the processing of palm nuts to palm oil, https://doi.org/10.1016/S0961-9534(02)00022-3, Biomass and Bioenergy, 22(6), 505-509 (2002).

Kaliyan, N., y Vance, R., Factors affecting strength and durability of densified biomass products, https://doi.org/10.1016/j.biombioe.2008.08.005, Biomass and Bioenergy, 33(3), 337-359 (2009).

Khlifi, S., Lajili, M., y otros cuatro autores, Briquettes Production from Olive Mill Waste under Optimal Temperature and Pressure Conditions: Physico-Chemical and Mechanical Characterizations, http://dx.doi.org/ 10.3390/en13051214, Energies, 13(5), 1214 (2020). 
MME, Ministerio de Minas y Energía., Atlas del Potencial Energético de la Biomasa Residual en Colombia, Bogotá, Colombia (2008).

Mitchual, S.J., Frimpong-Mensah, K., y Darkwa, N.A., Relationship between Physico-Mechanical Properties, Compacting Pressure and Mixing Proportion of Briquettes Produced from Maize Cobs and Sawdust, http://dx.doi.org/10.4236/jsbs.2014.41005, Journal of Sustainable Bioenergy Systems, 4, 50-60 (2014).

Moreno, M.C., Tratamiento de licor negro producto de la extracción de fibras del raquis de la palma de aceite africana mediante el proceso Foto-Fenton, Universidad de Los Andes, Bogotá, Colombia (2015).

Nasrin, A.B., Ma, A.N., y otros cinco autores, Oil palm biomass as potential substitution raw materials for commercial biomass briquettes production, https://doi.org/10.3844/ajassp.2008.179.183, American Journal of Applied Sciences, 5(3), 179-183 (2008).

Olorunnisola, A., Production of Fuel Briquettes from Waste Paper and Coconut Husk Admixtures, Agricultural Engineering International: the CIGR Ejournal, 9, 1-11 (2007).

Quintero, L., Buelvas, L., y Figueroa, O., Potencial energético de residuos agroindustriales para la producción de energía térmica del departamento del Cesar, Revista Agunkuya, 4(2), 44-57 (2014).

Quintero, L., Cantillo, O., y Robles, D., Análisis de la variabilidad del peso de los ladrillos y la importancia de estos parámetros para la producción en la ladrillera de las casitas, Valledupar - Cesar, https://doi.org/10.33132/27114260.1235, Revista Agunkuyáa, 8(1), 1-14 (2018).

Rey, C., Análisis espacial de la correlación entre cultivo de palma de aceite y desplazamiento forzado en Colombia, Cuadernos de Economía, 32(61), 683-718 (2013).

Ribó-Pérez, D., Herraiz-Cañete, A., y otros tres autores, Modelling biomass gasifiers in hybrid renewable energy microgrids; a complete procedure for enabling gasifiers simulation in HOMER, https://doi.org/10.1016/j.renene.2021.04.083, Renewable Energy, 174, 501-512 (2021).

Sierra-Vargas, F.E., Guerrero-Fajardo, C.A., y Mejía-Barragán, F., Determinación de la eficiencia de la cocción con leña en las veredas de Usme, Bogotá, Ingeniería Mecánica, 17(2), 185-194 (2014).

Wilaipon, P., Physical Characteristics of Maize Cob Briquette under Moderate Die Pressure, https://doi.org/10.3844/ajassp.2007.995.998, American Journal of Applied, 4(12), 995-998 (2007).

Yaman, S., Şahan, M., y otros tres autores, Production of fuel briquettes from olive refuse and paper mill waste, https://doi.org/10.1016/S0378-3820(00)00111-9, Fuel Processing Technology, 68(1), 23-31 (2000).

Zhang, J., y Guo, Y., Physical properties of solid fuel briquettes made from Caragana korshinskii Kom, https://doi.org/10.1016/j.powtec.2014.02.025, Powder Technology, 256, 293-299 (2014). 
